September 3, 2010

\title{
VAUGHT'S THEOREM ON AXIOMATIZABILITY BY A SCHEME
}

\author{
ALBERT VISSER
}

\begin{abstract}
In this paper we give an improvement of Vaught's Theorem on Axiomatizability by a Scheme. We show that any RE theory that directly interprets the theory of non-surjective unordered pairing, where pairing need not be functional, is axiomatizable by a scheme.
\end{abstract}

\section{INTRODUCTION}

Craig's well known trick shows us that every RE theory in predicate logic (of finite signature) has a decidable axiomatization. Inspection of the argument shows that we even get a p-time decidable axiomatization. It is less well known that there is a substantial class of theories that can be axiomatized by a scheme. This was shown by Vaught in his [Vau67]. Here is a precise formulation of the result. Consider the following axioms (in the language with $=$ and $\in$ ):

$\mathrm{vs}_{n} \vdash \forall x_{0}, \ldots, x_{n-1} \exists y \forall u\left(u \in y \leftrightarrow \bigvee_{i<n} u=x_{i}\right)$.

Note that vs $\mathrm{s}_{0}$ provides one or more empty sets, and that, for $0<k \leqslant n$, we have $\mathrm{vs}_{n} \vdash \mathrm{vs}_{k}$. The theory $\mathrm{VS}_{n}$ is axiomatized by $\mathrm{vs}_{0}, \ldots, \mathrm{vs}_{n}$, or, more economically, just by vs ${ }_{0}$ plus $\mathrm{vs}_{n}$. Vaught's Set Theory $\mathrm{VS}_{\omega}$, or simply VS, is the theory axiomatized by all the $\mathrm{vs}_{n}$.

We say that $U$ directly interprets $V$ iff there is an interpretation $K$ of $V$ in $U$, that is direct, i.e., that is not relativized and that translates identity to identity. We call a theory that directly interprets VS a Vaught theory. So, a Vaught theory contains a formula $A x y$ that satisfies all axioms of VS.

Vaught's theorem tells us that:

All Vaught theories are axiomatizable by a scheme.

Here are examples of Vaught theories of which it is not immediately evident how to axiomatize them by a scheme:

- $I \Delta_{0}+\left\{\Omega_{n} \mid n \in \omega\right\}$.

- $\mathrm{EA}+\left\{\operatorname{con}_{n}(\mathrm{EA}) \mid n \in \omega\right\}$ where EA is Elementary Arithmetic, aka Elementary Function Arithmetic or EFA, aka $I \Delta_{0}+$ Exp, and where 'con ${ }_{n}(\mathrm{EA})$ ' stands for consistency only involving proofs of complexity below $n$.

- $\mathrm{EA}+\left\{\operatorname{con}^{n}(\mathrm{EA}) \mid n \in \omega\right\}$, where $\operatorname{con}^{0}(E A):=\top, \operatorname{con}^{n+1}(E A):=\operatorname{con}\left(E A+\operatorname{con}^{n}(E A)\right)$.

- PRA (in a variant with finite signature).

We do not consider schemes with substitutions from restricted classes. Thus, $I \Delta_{0}$ and $S_{2}=I \Delta_{0}+\Omega_{1}$ are also examples of theories that are not evidently axiomatizable by a scheme.

2000 Mathematics Subject Classification. 03B10, 03B30, 03F25.

Key words and phrases. predicate logic, axiom, scheme. 
It is not difficult to see that VS interprets Tarski, Mostowski and Robinson's theory R. Hence, VS and therewith every consistent Vaught theory is essentially undecidable.

Note that $\mathrm{VS}_{2}$ is the theory of, not necessarily functional, non-surjective, unordered pairing. We call a theory that directly interprets $\mathrm{VS}_{2}$ : a pair theory. The main theorem of this paper is that:

All pair theories are axiomatizable by a scheme.

There are decidable pair theories. This is an immediate consequence of the results on ordered pairing by Richard Tenney ([Ten]), reported in [FR79], p162. A beautiful special case is the decidability of the true theory of the Cantor Pairing Function C with Successor, $\operatorname{Th}(\mathbb{N}, C, S)$, which is proved in [CR01]. Ferrante and Rackoff show that pair theories for functional ordered pairing are non-elementary. ${ }^{1}$ See [FR79]. The existence of decidable pair theories shows that our main theorem is a true improvement on Vaught's result. Ferrante and Rackoff's result shows that to be a pair theory is still a substantial restriction on theories.

Vaught provides several examples of RE theories that are not axiomatizable by a scheme. E.g., let $X$ be an RE, non-recursive set. Let $\mathrm{E} !_{n}$ be the statement, in the language of identity, that there are precisely $n$ elements. Let $U$ be the theory in the language of identity, axiomatized by the $\neg \mathrm{E} !_{n}$, for $n \in X$. If $U$ were axiomatizable by a scheme, then the class of finite models (say, as coded in the natural numbers in some standard way) would be decidable, since it is decidable whether a finite model satisfies a given scheme. Quod non.

At the end of our paper, we briefly discuss the possibility of compressing schemes to single sentences in the presence of comprehension principles.

Vaught's Paper. The present paper is in many respects a re-presentation or remake of Section 3 of Vaught's beautiful seven page paper [Vau67]. Vaught writes the following in the beginning of Section 3 about the improvement his Theorem 2, that states that any RE Vaught theory is axiomatizable by a scheme, makes over his Theorem 1. Vaught's theory $S$ is our theory VS. Vaught's theory $T_{1}$ is a theory of sequences and numbers.

Theorem 2 improves on Theorem 1 in two ways. For one thing, S appears to be somewhat weaker than $\mathrm{T}_{1}$ (as well as more elegant). More significant, however, is the fact that Theorem 2 states that $T$ is outright axiomatizable by a schema, rather than by a schema plus the axioms of $\mathrm{S}$.

Nevertheless, there do not appear to be any very interesting theories whose schematic axiomatizability is obtainable from Theorem 2 but not from Theorem 1. Consequently, we shall give a brief sketch of the proof.

It seems to me that the observation that Vaught's theorem extends to all theories that directly interpret a weak theory of pairing does extend the scope of the theorem sufficiently substantially to justify a more extended presentation.

Our proof follows Vaught's rather closely. Thus, it can be viewed for a large part as a careful exposition of Vaught's work. Here is the main difference. Vaught uses a very clever way of schematically axiomatizing expansions of VS with new predicates to insure that we need only one scheme without an infinity of auxiliary axioms. We, on the other hand, employ the fact that the theory of pairing locally

\footnotetext{
${ }^{1}$ I think that their result must also work in the non-extensional case, but did not try to prove this. Their result trivially transfers to functional unordered pairing.
} 
directly interprets the theory VS. Thus, we work with a varying interpretation of larger and larger parts of VS, where Vaught employs a fixed interpretation.

\section{Preliminaries}

In this paper, we consider RE theories of finite signature. These theories will one-sorted in most of the paper. The exception is Section 7, where we study theories with a second sort of classes. We will assume that our theories are officially relational, However, we will often pretend there are function symbols present. As is well known, the function symbols can be eliminated by an efficient translation.

Interpretations in this paper will be multi-dimensional relative interpretations with parameters. An interpretation $K$ of $U$ in $V$ is based on a translation $\tau$ of the language of $U$ to the language of $V$, such that, for all $U$-sentences $A$, we have $U \vdash A$ implies $V \vdash A^{\tau}$. See e.g. [Vis06] or [Vis09b] or [Vis08] for careful definitions. An interpretation is direct iff it is unrelativized and does translate identity into identity. Note that direct interpretations are automatically one-dimensional. A theory $V$ locally interprets a theory $U$ iff $V$ interprets every finite subtheory of $U$. We remind the reader of the notations:

- $V \triangleright U$ for: $V$ interprets $U$.

- $U \triangleleft V$ for: $U$ is interpretable in $V$.

Further determinations of the kind of interpretability intended will be added as subscript. E.g., $V \triangleright_{\text {dir }} U$ will mean: $V$ directly interprets $U$.

A scheme $\mathcal{S}$ for a theory $U$ is obtained as follows. We extend the signature, say $\Sigma_{U}$ of $U$ with a finite number of predicate symbols to a new signature $\Sigma_{U}^{+}$. We assume that the new symbols are marked in $\Sigma_{U}^{+}$. A scheme is a sentence in the extended language. Let's say that an s-translation is a translation of the language given by $\Sigma_{U}^{+}$into the language of $U$ that is constant on the non-marked or old vocabulary; the predicate symbols of the new vocabulary are replaced by formulas of the old vocabulary. We do not have domain restriction in s-translations. The scheme $\mathcal{S}$ is valid in $U$ if, for all s-translations $\tau, U \vdash \mathcal{S}^{\tau}$. Our formulations allow two readings: do we allow parameters or not in the translations $\tau$. I will assume that the default reading of scheme is scheme with parameters. This means that the s-translations in the definition of validity range over translations with parameters. These parameters are unrestricted, i.e., they range over the full domain of $U$, if we do not allow parameters, we will speak about a closed scheme.

\section{Theories of Number(s)}

We formulate theories that can be a both theories of a number and of all numbers. The theory $\mathrm{TN}_{0}$ is intended to be possibly the theory of a number. It is given as follows. We have, apart from equality, the following symbols in the signature: a constant 0 , two binary relation symbols $\mathbf{S}$ and $\leqslant$, and two ternary relations $\mathrm{A}$ and M. The theory is axiomatized as follows.

$$
\begin{aligned}
& \operatorname{tn} 1 \vdash(\mathrm{S} x y \wedge \mathrm{S} u v) \rightarrow((x=u \wedge y=v) \vee(x \neq u \wedge y \neq v)), \\
& \operatorname{tn} 2 \vdash \neg \mathrm{S} x 0, \\
& \operatorname{tn} 3 \vdash x=0 \vee \exists y \mathrm{~S} y x \\
& \operatorname{tn} 4 \vdash(\mathrm{A} x y u \wedge \mathrm{A} x y v) \rightarrow u=v, \\
& \operatorname{tn} 5 \vdash \mathrm{A} x 0 x, \\
& \operatorname{tn} 6 \vdash \exists u(\mathrm{~S} y u \wedge \mathrm{A} x u v) \leftrightarrow \exists z(\mathrm{~S} z v \wedge \mathrm{A} x y z),
\end{aligned}
$$




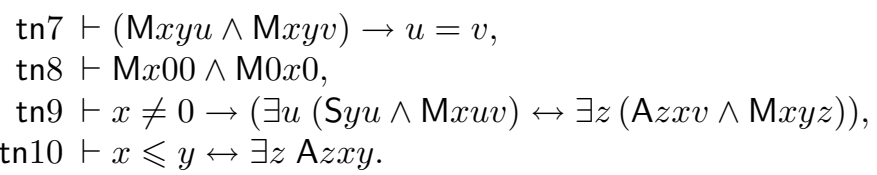

The assumption of Axiom tn9 is essential. To see that, suppose we drop it. Since we have A000 and M0y0, it would follow that, for some $u$, Syu. This frustrates our intention to formulate a theory that can also be the theory of a number. Note that if we would both drop the assumption of Axiom tn9 and the second conjunct of $\operatorname{tn} 8$, we still would be able to prove the undesirable: $\exists x_{1}, \ldots, x_{n}\left(S 0 x_{1} \wedge \ldots \wedge S x_{n-1} x_{n}\right)$, for every $n>0$.

We will use partial function notation. Partial terms will be eliminated via the algorithm $(\cdot)^{\star}$. We adopt the small scope convention and we employ weak evaluation for multiplication with 0 . We first translate formulas of the form $t=x$, where $x$ does not occur in $t$.

- $(y=x)^{\star}:=(y=x)$,

- $(0=x)^{\star}:=(0=x)$,

- $(\mathrm{S} t=x)^{\star}:=\exists x_{0}\left(\left(t=x_{0}\right)^{\star} \wedge \mathrm{S} x_{0} x\right)$,

- $\left(t_{0}+t_{1}=x\right)^{\star}:=\exists x_{0} \exists z\left(\left(t_{0}=x_{0}\right)^{\star} \wedge\left(t_{1}=x_{1}\right)^{\star} \wedge \mathrm{A} x_{0} x_{1} x\right)$,

- $\left(t_{0} \times t_{1}=x\right)^{\star}=\left(\exists x_{0}\left(\left(t_{0}=x_{0}\right)^{\star} \wedge x_{0}=0 \wedge x=0\right) \vee\right.$ $\left(\exists x_{1}\left(\left(t_{1}=x_{1}\right)^{\star} \wedge x_{1}=0 \wedge x=0\right) \vee\right.$ $\left.\exists x_{0} \exists x_{1}\left(\left(t_{0}=x_{0}\right)^{\star} \wedge\left(t_{1}=x_{1}\right)^{\star} \wedge \mathrm{M} x_{0} x_{1} x\right)\right)$.

We now define the full translation for all formulas not of the form $t=x$, where $x$ is not in $t$, as follows:

- $\left(t_{0}=t_{1}\right)^{\star}:=\left(\exists x_{0} \exists x_{1}\left(\left(t_{0}=x_{0}\right)^{\star} \wedge\left(t_{1}=x_{1}\right)^{\star} \wedge x_{0}=x_{1}\right)\right)$,

- $\left(t_{0} \leqslant t_{1}\right)^{\star}:=\left(\exists x_{0} \exists x_{1}\left(\left(t_{0}=x_{0}\right)^{\star} \wedge\left(t_{1}=x_{1}\right)^{\star} \wedge x_{0} \leqslant x_{1}\right)\right)$,

- $(\cdot)^{\star}$ commutes with the propositional connectives and quantifiers.

We use $t \simeq u$ for: either $t$ and $u$ are both defined and their values are equal, or both are undefined. The expression $t \downarrow$ will mean: $t$ is defined. The expression $t \uparrow$ will mean: $t$ is undefined. We will use + for $A$ and $\times$ or $\cdot$ for $M$. In these notations we have the following consequences of our axioms:

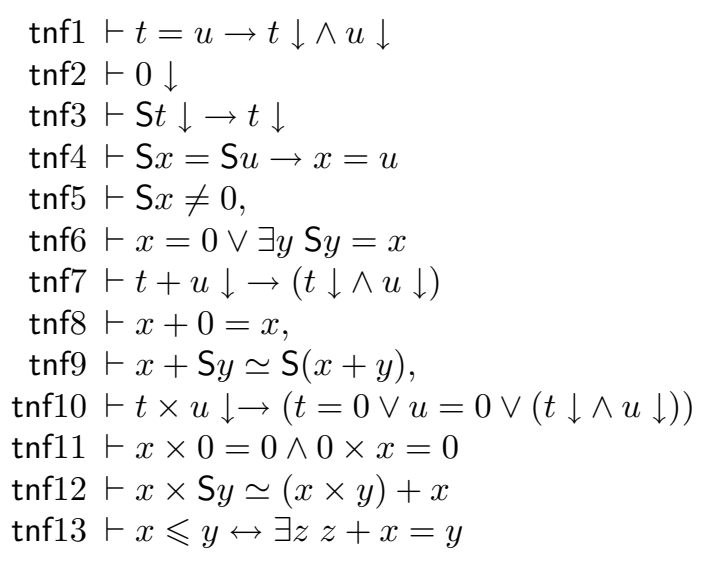

It is easy to see that these principles follow indeed from the axioms. Note that to prove tnf12, we need to split into the cases whether $x$ is or is not 0 and whether $y$ is or is not 0 . The antecedent of tn9 disappears in tnf12, since our conventions do 
not demand the successor to be defined in that case. Note that, conversely, we can derive our original axioms from the new ones except $\operatorname{tn} 8$ and part of $\operatorname{tn} 9$.

The disappearance of the assumption of $\operatorname{tn} 9$ is the main point of the use of the 'weak convention'. It allows us to compute with undefined terms in the usual way, giving us e.g. Lemma 3.1(4).

We write $\underline{n}$ for $\mathrm{S}^{n} 0$. The theory $\mathrm{TN}_{n}$ is $\mathrm{TN}_{0}+\underline{n} \downarrow$. The theory $\mathrm{TN}_{\omega}$ is the union of the $\mathrm{TN}_{n}$.

Consider any natural number $n$. We define $0, \mathrm{~S}, \mathrm{~A}, \mathrm{M}, \leqslant$ on $\{0, \cdots, n\}$ in the obvious way, e.g., Axyz:↔x+y=z and $x, y, z \leqslant n$. We call this model $\mathbb{N}_{n}$. We have $\mathbb{N}_{n} \models \mathrm{TN}_{n}$. Of course, we also have $\mathbb{N} \models \mathrm{TN}_{\omega}$.

Here is a lemma collecting some basic facts about the $\mathrm{TN}_{n}$.

Lemma 3.1. (1) The relation $\simeq$ is $\mathrm{TN}_{0}$-provably an equivalence relation between terms.

(2) Suppose $t$ and $t^{\prime}$ are substitutable for $x$ in $A x$, then:

$\mathrm{TN}_{0} \vdash t \simeq t^{\prime} \rightarrow\left(A t \leftrightarrow A t^{\prime}\right)$

(3) Suppose $t \vec{m}=n$. Then, $\mathrm{TN}_{0} \vdash t \underline{\vec{m}} \simeq \underline{n}$.

(4) Suppose $t \vec{m}=t^{\prime} \vec{m}$. Then, $\mathrm{TN}_{0} \vdash t \underline{m} \simeq t^{\prime} \underline{\vec{m}}$.

(5) Suppose $t \vec{m} \leqslant t^{\prime} \vec{m}$. Then, $\mathrm{TN}_{0} \vdash t^{\prime} \underline{\vec{m}} \downarrow \rightarrow t \underline{m} \leqslant t^{\prime} \underline{\vec{m}}$.

(6) If $t \vec{m} \neq t^{\prime} \vec{m}$, then $\mathrm{TN}_{0} \vdash t \underline{\vec{m}} \neq t^{\prime} \underline{\vec{m}}$.

(7) If $t^{\prime} \vec{m}<t \vec{m}$, then $\mathrm{TN}_{0} \vdash \neg t \underline{\underline{\vec{m}}} \leqslant t^{\prime} \underline{\vec{m}}$.

(8) Suppose $t \vec{m}=t^{\prime} \vec{m} \leqslant n$. Then, $\mathrm{TN}_{n} \vdash t \underline{\vec{m}}=t^{\prime} \underline{\vec{m}}$.

(9) Suppose $t \vec{m} \leqslant t^{\prime} \vec{m} \leqslant n$. Then $\mathrm{TN}_{n} \vdash t \overrightarrow{\vec{m}} \leqslant t^{\prime} \overrightarrow{\vec{m}}$.

(10) $\mathrm{TN}_{0} \vdash \bigvee_{m<n} x=\underline{m} \vee \underline{n} \leqslant x$.

(11) $\mathrm{TN}_{0} \vdash(\underline{n} \uparrow \vee x \leqslant \underline{n}) \leftrightarrow \bigvee_{m \leqslant n} x=\underline{m}$.

(12) $\mathrm{TN}_{n} \vdash x \leqslant \underline{n} \vee \underline{n} \leqslant x$.

(13) $\mathrm{TN}_{0} \vdash(x \leqslant \underline{n} \wedge \underline{n} \leqslant x) \rightarrow x=\underline{n}$.

(14) $\mathrm{TN}_{0} \vdash(\underline{n} \leqslant x \wedge x \leqslant y) \rightarrow \underline{n} \leqslant y$.

(15) $\mathrm{TN}_{0} \vdash(x \leqslant \underline{n} \wedge \underline{n} \leqslant y) \rightarrow x \leqslant y$.

(16) $\mathrm{TN}_{0} \vdash(x \leqslant \bar{y} \wedge \bar{y} \leqslant \underline{n}) \rightarrow x \leqslant \underline{n}$.

Proof. We prove (10) by induction on $n$. The case that $n=0$ is easy. Suppose $n=k+1$ and we have $\mathrm{TN}_{0} \vdash \bigvee_{m<k} x=\underline{m} \vee \underline{k} \leqslant x$. Reason in $\mathrm{TN}_{0}$. If we have $\bigvee_{m<k} x=\underline{m}$, then we also have $\bigvee_{m<n} x=\underline{m}$ and we are done. Suppose we have $\underline{k} \leqslant x$. So, for some $u, u+\underline{k}=x$. In case $u=0$, we find $0+\underline{k}=x$ and hence, by an easy argument, $\underline{k}=x$ and, thus, $\bigvee_{m<n} x=\underline{m}$. In case $u=\mathrm{S} v$, we find $\mathrm{S} v+\underline{k}=x$. By an easy argument this gives $v+\underline{n}=x$, so $\underline{n} \leqslant x$.

Lemma 3.2. Suppose $A \vec{x}$ is a $\Delta_{0}$-formula. There is a term $t_{A} \vec{x}$, which majorizes every $x$ in $\vec{x}$, such that, for every $n$ and for every $\vec{m}$ with $t_{A} \vec{m} \leqslant n$, we have:

(1) If $A \vec{m}$, then $\mathrm{TN}_{n} \vdash A \underline{\vec{m}}$,

(2) If $\neg A \vec{m}$, then $\mathrm{TN}_{n} \vdash \neg A \underline{\vec{m}}$.

Proof. The proof is by induction, simultaneous in both cases, on the complexity of $A$. Suppose $A$ is $t \vec{x}=t^{\prime} \vec{x}$. Then, we can take $t_{A} \vec{x}:=t \vec{x}+t^{\prime} \vec{x}$. The cases of the Boolean connectives are obvious.

Suppose $A \vec{x}$ is $\forall y \leqslant t \vec{x} B \vec{x} y$. We take $t_{A} \vec{x}:=t \vec{x}+t_{B}(\vec{x}, t \vec{x})$. Suppose $t_{A} \vec{m} \leqslant n$. Let $t \vec{m}=k$. Since, $t \vec{m} \leqslant n$, we find, by Lemma 3.1(8), $\operatorname{TN}_{n} \vdash t \underline{\vec{m}}=\underline{k}$. By 
Lemma 3.1(11), we find:

$$
\mathrm{TN}_{n} \vdash A \vec{m} \leftrightarrow \bigwedge_{p \leqslant k} B(\underline{\vec{m}}, \underline{p}) .
$$

Since, $t_{B}(\vec{m}, p) \leqslant t_{B}(\vec{m}, k) \leqslant t_{A}(\vec{m}) \leqslant n$, the desired result is immediate from the Induction Hypothesis. The case of the bounded existential quantifier is similar.

Suppose $S \vec{x}$ is a $\Sigma_{1}^{0}$-formula. Say, $S \vec{x}=\exists \vec{y} S_{0} \vec{x} \vec{y}$, where $S_{0} \vec{x} \vec{y}$ is $\Delta_{0}$. We define $S^{z} \vec{x}$, by $\exists \vec{y} \leqslant z\left(t_{S_{0}} \vec{x} \vec{y} \leqslant z \wedge S_{0} \vec{x} \vec{y}\right)$. Here $t_{S_{0}}$ is the term provided by Lemma 3.2.

Lemma 3.3. Let $S \vec{x}$ be a $\Sigma_{0}$-formula.

(1) We have: $S \vec{m}$ iff, for some $n, S^{n} \vec{m}$.

(2) Suppose $S^{n} \vec{m}$. Then, $\mathrm{TN}_{n} \vdash S \underline{\underline{n}} \underline{\vec{m}}$.

(3) Suppose $\neg S^{n} \vec{m}$. Then, $\mathrm{TN}_{0} \vdash \neg S^{\underline{n}} \underline{\vec{m}}$.

Proof. We treat (2). Suppose $S^{n} \vec{m}$. So, for some $\vec{k}$, we have $t_{S_{0}} \vec{m} \vec{k} \leqslant n$ and $S_{0} \vec{m} \vec{k}$. By Lemma 3.1(9), $\operatorname{TN}_{n} \vdash t_{S_{0}}(\underline{\vec{m}}, \underline{\vec{k}}) \leqslant \underline{n}$. By Lemma 3.2, we have $\operatorname{TN}_{n} \vdash S_{0}(\underline{\vec{m}}, \underline{\vec{k}})$. Hence, $\mathrm{TN}_{n} \vdash S^{n} \underline{\underline{\vec{m}}}$.

We treat (3). Suppose $\neg S^{n} \vec{m}$. Let $U:=\mathrm{TN}_{0}+S \underline{\underline{n}} \underline{\vec{m}}$. Note that $U$ extends $\mathrm{TN}_{n}$. By Lemma 3.2, we find, for $\vec{k}$ such that $t_{S_{0}} \vec{m} \vec{k} \leqslant n$, that $U \vdash \neg S_{0}(\underline{\vec{m}}, \underline{\vec{k}})$. Also, by Lemma 3.1(7), if $n<t_{S_{0}} \vec{m} \vec{k} \leqslant n$, then $U \vdash \neg t_{S_{0}}(\underline{\vec{m}}, \underline{\vec{k}}) \leqslant \underline{n}$. By Lemma 3.1(11), we find $U \vdash \bigvee_{\vec{k}<n}\left(t_{S_{0}}(\underline{\vec{m}}, \underline{\vec{k}}) \leqslant \underline{n} \wedge S_{0}(\underline{\vec{m}}, \underline{\vec{k}})\right)$. Combining, we find $U \vdash \perp$. So, we may conclude that $\mathrm{TN}_{0} \vdash \neg S^{n} \vec{m}$.

Lemma 3.4. Suppose $S(\vec{x}, y)$ is a $\Sigma_{1}^{0}$-formula representing a function. Then there is a $\Sigma_{1}^{0}$-formula $S^{\circ}(\vec{x}, y)$, such that, for all $\vec{m}$, if $S(\vec{m}, n)$, then, for some $k$, $\mathrm{TN}_{k} \vdash S^{\circ}(\vec{m}, n) \wedge \forall y\left(S^{\circ}(\underline{\vec{m}}, y) \rightarrow \underline{n}=y\right)$.

Proof. Suppose $S(\vec{x}, y)$, is $\exists \vec{z} S_{0} \vec{x} y \vec{z}$, where $S_{0}$ is in $\Delta_{0}$. Let:

- $S_{0}^{\circ}(\vec{x}, y, z): \leftrightarrow y \leqslant z \wedge \exists \vec{u} \leqslant z S_{0} \vec{x} y \vec{u} \wedge \forall \vec{v}, w \leqslant z\left(S_{0} \vec{x} w \vec{v} \rightarrow w=y\right)$.

- $S^{\circ}(\vec{x}, y): \leftrightarrow \exists z S_{0} \vec{x} y z$.

Suppose $S \vec{m} n$. By the functionality of $S$, we can clearly find a $q$ such that $S_{0}^{\circ} \vec{m} n q$. Let $k \geqslant t_{S_{0}^{\circ}} \vec{m} n q$. By Lemma 3.2 , we have $\operatorname{TN}_{k} \vdash S_{0}^{\circ}(\underline{\vec{m}}, \underline{n}, \underline{q})$.

We reason in $\mathrm{TN}_{k}$. Suppose $S_{0}^{\circ}(\underline{\vec{m}}, y, z)$. By Lemma 3.1(12), either $z \leqslant \underline{q}$ or $\underline{q} \leqslant z$.

Suppose $z \leqslant q$. Since $S_{0}^{\circ}(\vec{m}, n, q)$, for every $\vec{v}, w \leqslant q$, if $S_{0}(\underline{\vec{m}}, w, \vec{v})$, then $w=\underline{n}$. On the other hand, by $S_{0}^{\circ}(\underline{\vec{m}}, y, z)$, we have $y \leqslant z$ and, for some $\vec{u} \leqslant z, S_{0}(\underline{\vec{m}}, y, \overrightarrow{\vec{u}})$. By Lemma $3.1(16)$, we find $\vec{u}, y \leqslant \underline{q}$. So, it follows that $y=\underline{n}$.

Suppose $q \leqslant z$. Since $S_{0}^{\circ}(\underline{\vec{m}}, y, z)$, for every $\vec{v}, w \leqslant z$, if $S_{0}(\underline{\vec{m}}, w, \vec{v})$, then $w=y$. On the other hand, by $S_{0}^{\circ}(\underline{\vec{m}}, \underline{n}, q)$, we have $\underline{n} \leqslant q$ and, for some $\vec{u} \leqslant q, S_{0}(\underline{\vec{m}}, n, \vec{u})$. Вy Lemma 3.1(15), we find $\vec{u}, \underline{n} \leqslant z$. So, $\underline{n}=y$.

Lemma 3.5 (Gödel Fixed Point Lemma). Suppose that $K$ is an interpretation of $\mathrm{TN}_{0}$ in $U$. Let $A x \vec{y}$ be a formula in the language of $U$. Suppose that:

$$
U \vdash A x \vec{y} \rightarrow \delta_{K}(x) \text { and } U \vdash x_{0}={ }_{K} x_{1} \rightarrow\left(A x_{0} \vec{y} \leftrightarrow A x_{1} \vec{y}\right) .
$$

Then, there is a formula $B \vec{v}$ and an $n$, such that

$$
U+(\underline{n} \downarrow)^{K} \vdash B \vec{z} \leftrightarrow \exists x\left((x=\ulcorner B \vec{v}\urcorner)^{K} \wedge A x \vec{z}\right) .
$$


Proof. Suppose sub is a $\Sigma_{1}^{0}$-representation of the Gödel substitution function or the language of $U$, such that $\operatorname{sub}\left(n,\left\ulcorner C x \vec{y},\left\ulcorner\exists x: \delta_{K}\left((x=\underline{n})^{K} \wedge C x \vec{y}\right)\right.\right.\right.$, where $x$ is a designated variable. Let:

- $A^{*} y \vec{v}: \leftrightarrow \exists x: \delta_{K}\left(\left(\operatorname{sub}^{\circ}(y, y, x)\right)^{K} \wedge A x \vec{v}\right)$,

- $m:=\left\ulcorner A^{*} u \vec{v}\right\urcorner$,

- $B \vec{v}: \leftrightarrow \exists y: \delta_{K}\left((\underline{m}=x)^{K} \wedge A^{*} y \vec{v}\right)$.

- $k:=\ulcorner B \vec{v}\urcorner$.

By Lemma 3.4, we can find $n$ such that:

$$
\operatorname{TN}_{n} \vdash \operatorname{sub}^{\circ}(\underline{m}, \underline{m}, \underline{k}) \wedge \forall y\left(\operatorname{sub}^{\circ}(\underline{m}, \underline{m}, y) \rightarrow \underline{k}=y\right) .
$$

Thus, we have, in $U+(\underline{n} \downarrow)^{K}$ :

$$
\begin{aligned}
B \vec{z} & \leftrightarrow \exists y: \delta_{K}\left((\underline{m}=y)^{K} \wedge \exists x: \delta_{K}\left(\left(\operatorname{sub}^{\circ}(y, y, x)\right)^{K} \wedge A x \vec{z}\right)\right) \\
& \leftrightarrow \exists x, y: \delta_{K}\left(\left(\underline{m}=y \wedge \operatorname{sub}^{\circ}(y, y, x)\right)^{K} \wedge A x \vec{z}\right) \\
& \leftrightarrow \exists x: \delta_{K}\left((\underline{k}=x)^{K} \wedge A x \vec{z}\right)
\end{aligned}
$$

\section{SETS}

We define a theory of sets $\mathrm{TS}_{0}$ as follows. Our signature consists of two unary symbols $\mathrm{N}$, Z, five binary symbols $\mathrm{S}, \leqslant, \mathrm{E}, \in, \eta$, and two ternary symbols $\mathrm{A}$ and $\mathrm{M}$. The intended interpretation of $\eta$ is the relation between a set and its cardinality. However, our axioms do not go far in constraining $\eta$ to be so.

We let $a, b, c, \ldots$ range over $\mathrm{N}$. This means that if e.g. ' $a$ ' occurs freely in a formula, then we assume that $\mathrm{N}(a)$ is present in the context. The formula $\exists a A$ means $\exists a(\mathrm{~N} a \wedge A)$, and similarly for the universal quantifier. We use $x, y, z, w, \ldots$ to range over all objects.

ts1 Axioms stating that $\mathrm{Z}, \mathrm{E}, \mathrm{S}, \leqslant, \mathrm{A}, \mathrm{M}$ are relations on N. E.g.,

$\vdash \mathrm{A} x y z \rightarrow(\mathrm{N} x \wedge \mathrm{N} y \wedge \mathrm{N} z)$.

An axiom stating that $\eta$ is a relation on $\mathrm{N}$ in the second component, i.e., $\vdash x \eta y \rightarrow \mathrm{N} y$,

ts2 Axioms stating that $\mathrm{E}$ is an equivalence relation of $\mathrm{N}$ which is a congruence w.r.t. Z, S, $\leqslant, A, M$. An axiom stating that $E$ is a congruence w.r.t $\eta$ in the second component, i.e., $\vdash(x \eta a \wedge a \mathrm{E} b) \rightarrow x \eta b$,

ts3 $\vdash \exists a \mathrm{Z} a$,

$\mathrm{ts} 4 \vdash(\mathrm{Z} a \wedge \mathrm{Z} b) \rightarrow a \mathrm{E} b$,

ts $5 \vdash(\mathrm{S} a b \wedge \mathrm{S} c d) \rightarrow((a \mathrm{E} c \wedge b \mathrm{E} d) \vee(\neg a \mathrm{E} c \wedge \neg b \mathrm{E} d))$,

ts6 $\vdash \neg(\mathrm{S} a b \wedge \mathrm{Z} b)$,

ts7 $\vdash \mathrm{Z} a \vee \exists b \mathrm{~S} b a$

ts $8 \vdash(\mathrm{A} a b c \wedge \mathrm{A} a b d) \rightarrow c \mathrm{E} d$,

ts9 $\vdash \mathrm{Z} b \rightarrow \mathrm{A} a b a$,

ts10 $\vdash \exists d(\mathrm{~S} b d \wedge \mathrm{A} a d e) \leftrightarrow \exists c(\mathrm{~S} c e \wedge \mathrm{A} a b c)$,

$\mathrm{ts} 11 \vdash(\mathrm{M} a b c \wedge \mathrm{M} a b d) \rightarrow c \mathrm{E} d$,

ts12 $\vdash \mathrm{Z} b \rightarrow(\mathrm{M} a b b \wedge \mathrm{M} b a b)$,

$\mathrm{ts} 13 \vdash \neg \mathrm{Z} a \rightarrow(\exists d(\mathrm{~S} b d \wedge \mathrm{M} a d e) \leftrightarrow \exists c($ Acae $\wedge \mathrm{M} a b c))$,

ts14 $\vdash a \leqslant b \leftrightarrow \exists c$ Acab.

ts $15 \vdash \mathrm{Z} a \rightarrow \exists x(x \eta a \wedge \forall y y \notin x)$,

ts16 $\vdash(x \eta a \wedge \mathrm{S} a b) \rightarrow \forall u \exists y(y \eta b \wedge \forall v(v \in y \leftrightarrow(v \in x \vee v=u)))$ 
Note that our first 14 axioms are just translations of the $\mathrm{TN}_{0}$ axioms. So, modulo translation, our new theory is the extension of $\mathrm{TN}_{0}$ with two axioms. Let us define:

- $\operatorname{empty}(x): \leftrightarrow \forall y y \notin x$,

- $\operatorname{adj}_{u}(x, y): \leftrightarrow \forall v(v \in y \leftrightarrow(v \in x \vee v=u))$,

With these definitions, our new axioms read:

- $\vdash \mathrm{Z} a \rightarrow \exists x(x \eta a \wedge \operatorname{empty}(x))$,

- $\vdash(x \eta a \wedge \mathrm{S} a b) \rightarrow \forall u \exists y\left(y \eta b \wedge \operatorname{adj}_{u}(x, y)\right)$.

Par abus de langage, we suppress the standard interpretation of $\mathrm{TN}_{0}$ in $\mathrm{TS}_{0}$. We define $\mathrm{TS}_{n}:=\mathrm{TS}_{0}+\underline{n} \downarrow$

Theorem 4.1. The theory $\mathrm{TS}_{n}$ is directly interpretable in $\mathrm{VS}_{2}$ via a one dimensional interpretation without parameters. If follows that $\mathrm{TS}_{\omega}$ is locally directly interpretable in $\mathrm{VS}_{2}$.

Proof. We fix some $k$ be such that $2^{k} \geqslant n$. We define:

- $x \in^{0} y: \leftrightarrow x=y, x \in^{j+1} y: \leftrightarrow \exists z\left(x \in z \wedge z \in^{j} y\right)$.

- $\operatorname{set}(y): \leftrightarrow \forall u_{0}, \ldots, u_{n}\left(\bigwedge_{j \leqslant n} u_{i} \in^{k} y \rightarrow \bigvee_{i<j \leqslant n} u_{i}=u_{j}\right)$

- For $j \leqslant n$, $j(x): \leftrightarrow \operatorname{set}(x) \wedge \exists u_{0}, \ldots u_{j-1}\left(\bigwedge_{i<j} u_{i} \in x \wedge\right.$$$
\left.\bigwedge_{s<i<j} u_{s} \neq u_{i} \wedge \forall v \in y \bigvee_{i<j} v=u_{i}\right) .
$$

Let $k$ be such that $2^{k} \geqslant n$. We define a direct translation $\tau$ as follows.

- $\mathrm{N}_{\tau}(x): \leftrightarrow x=x$,

- $\mathrm{Z}_{\tau} x: \leftrightarrow 0(x)$,

- $\mathrm{S}_{\tau} x y: \leftrightarrow \bigvee_{j<n}(j(x) \wedge(j+1) y)$,

- $x \leqslant_{\tau} y: \leftrightarrow \bigvee_{i \leqslant j \leqslant n}(i(x) \wedge j(y))$,

- $x \mathrm{E}_{\tau} y: \leftrightarrow \bigvee_{i \leqslant n}(i(x) \wedge i(y))$,

- $x \in_{\tau} y: \leftrightarrow x \in^{k} y \wedge \operatorname{set}(y)$,

- $x \eta_{\tau} y: \leftrightarrow x \in_{\tau} y$,

- $\mathrm{A}_{\tau} x y z: \leftrightarrow \bigvee_{s+i=j \& j \leqslant n}(s(x) \wedge i(y) \wedge j(z))$,

- $\mathrm{M}_{\tau} x y z: \leftrightarrow \bigvee_{s \times i=j \& s, i, j \leqslant n}(s(x) \wedge i(y) \wedge j(z))$,

Note that in $\mathrm{VS}_{2}$ we can prove that there are infinitely many objects, by considering iterated singletons on empty sets. So we can show in $\mathrm{VS}_{2}$ that $n$ (as interpreted by $\tau$ ) exists. E.g., in case $n=7$, we produce singletons $a_{0}, \ldots, a_{6}$, where $a_{i} \in a_{i+1}$. We may show that these are pairwise distinct. Next we find $b_{0}, \ldots, b_{3}$, where $b_{0}$, $b_{1}, b_{2}$ are unordered pairs, $b_{3}$ is a singleton and $a_{0}, a_{1} \in b_{0}, a_{2}, a_{3} \in b_{1}, a_{4}, a_{5} \in b_{2}$ and $a_{6} \in b_{3}$. Note that the $b_{i}$ are pairwise distinct. Next we find unordered pairs $c_{0}$ and $c_{1}$ such that $b_{0}, b_{1} \in c_{0}$ and $b_{2}, b_{3} \in c_{1}$. Note that $c_{0} \neq c_{1}$, Finally, we find an unordered pair $d$ with $c_{0}, c_{1} \in d$. The object $d$ will be a set with 7 elements under $\tau$. Using these ideas we may easily show that $\tau$ yields an interpretation of $\mathrm{TS}_{n}$ in $\mathrm{VS}_{2}$.

Remark 4.2. The interpretation constructed in the preceding proof is highly inefficient in terms of the size of the interpreting formulas as a function of $n$. Employing the usual definitions of set theory we can interpret $\mathrm{TS}_{n}$ in far more efficient ways. Also, we can do much better in defining $\in^{k}$. Such efficiency could be necessary if we want to verify our theorem in $S_{2}^{1}$. Also, it would be quite interesting to try to adapt our methods to reprove the lower bound result of Ferrante and Rackoff (see 
[FR79]). This would also ask for more efficient interpretations. However, for our present purposes such efficiency is not needed.

\section{Satisfaction}

Consider any theory $U$. We extend the language of $U$ with the vocabulary of $\mathrm{TS}_{0}$ plus a new binary predicate sat. We define the formula SAT that says roughly that 'below $c$ ' the predicate sat behaves as a satisfaction predicate with the first argument in the role of assignment and the second argument in the role of formula. To keep things readable we assume that the language of $U$ has a unary predicate $P$, a binary predicate $Q$ and that the only propositional connectives are the 0 -ary connectives $T$ and $\perp$, negation and conjunction and that the only quantifier is universal quantification.

We may find $\Sigma_{1}^{0}$-formulas defining some useful predicates:

- $\operatorname{var}(b):$ ' $b$ codes a variable'.

- $\mathrm{fv}(b, a)$ : ' $a$ codes a formula and $b$ codes a free variable occurring in $a$.'

- $\llbracket P \rrbracket(b, a)$ : ' $b$ codes a variable ' $v$ ' and $a$ codes ' $P v$ '.' Similarly for $\llbracket Q \rrbracket(b, a)$.

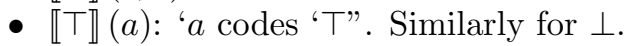

- $\llbracket \neg \rrbracket(b, a)$ : ' $b$ codes a formula $A$ and $a$ codes $\neg A$.'

- The other cases are as expected.

We need some further definitions:

- $x \subseteq y: \leftrightarrow \forall z(z \in x \rightarrow z \in y)$,

- $\operatorname{pair}(x, y, z): \leftrightarrow \exists u, v \forall w((w \in z \leftrightarrow(w=u \vee w=v)) \wedge$

- $\operatorname{rel}(x): \leftrightarrow \forall y \in x \exists u, v \operatorname{pair}(u, v, y)$

$(w \in u \leftrightarrow w=x) \wedge(w \in v \leftrightarrow(w=x \vee w=y)))$

- $\operatorname{fun}(x): \leftrightarrow \forall y, z \in x \forall u, v, w((\operatorname{pair}(u, v, y) \wedge \operatorname{pair}(u, w, z)) \rightarrow y=z) .^{2}$

- $x(y)=z: \leftrightarrow$ fun $(x) \wedge \exists u \in x \operatorname{pair}(y, z, u)$

- $\operatorname{dom}(y, x): \leftrightarrow \exists z x(y)=z$

- $\operatorname{funn}(x): \leftrightarrow \operatorname{fun}(x) \wedge \forall y(\operatorname{dom}(y, x) \rightarrow \mathrm{N}(y)) \wedge$ $\forall a, b, u, v((a \mathrm{E} b \wedge x(a)=u \wedge x(b)=v) \rightarrow(a=b \wedge u=v))$

- $x \star a=y: \leftrightarrow \operatorname{funn}(x) \wedge \exists b(a \mathrm{E} b \wedge x(b)=y)$

- $\operatorname{ass}(c, x, a): \leftrightarrow \operatorname{funn}(x) \wedge \forall b\left(\operatorname{dom}(b, x) \leftrightarrow \operatorname{var}^{c}(b)\right)$

- $x[a] y: \leftrightarrow \operatorname{funn}(x) \wedge \operatorname{funn}(y) \wedge \forall b(\neg a \mathrm{E} b \rightarrow x \star b \simeq y \star b) \wedge y \star a \downarrow)$

The definition of the 'this is a satisfaction predicate below c'-predicate SAT is given in Figure 5.

Theorem 5.1. Suppose we have an interpretation $K: U \triangleright_{\mathrm{dir}} \mathrm{TS}_{0}$. We will suppress ' $K$ ' notationally. Let $A$ be $A\left(v_{0}, \ldots v_{k-1}\right)$. Suppose we have form ${ }^{n}(A)$. We assume that $n \geqslant 2$. We have:

$$
U+\underline{n} \leqslant b+\operatorname{SAT}(b, B)+\operatorname{ass}(b, x,\ulcorner A\urcorner) \vdash A\left(x \star\left\ulcorner v_{0}\right\urcorner, \ldots, x \star\left\ulcorner v_{i}\right\urcorner\right) \leftrightarrow B(x,\ulcorner A\urcorner) .
$$

Proof. We proceed by induction on $A$. We treat the case that $A=\forall v C$, where $C$ is $C\left(v, v_{0}, \ldots, v_{k-1}\right)$.

We have form ${ }^{n}(\ulcorner A\urcorner)$ and, hence, form ${ }^{n}(\ulcorner C\urcorner), \llbracket \forall \rrbracket^{n}(\ulcorner v\urcorner,\ulcorner C\urcorner,\ulcorner A\urcorner)$ and $\mathrm{fv}^{n}(\ulcorner x\urcorner)$. Let $V$ be the theory $U+\underline{n} \leqslant b+\operatorname{SAT}(b, B)+\operatorname{ass}(b, x,\ulcorner A\urcorner)$. In $V$, we have form ${ }^{b}(\ulcorner A\urcorner)$,

\footnotetext{
${ }^{2}$ We have ' $y=z$ ' rather than ' $v=w$ ' in the consequent, because our pairing is non-functional. To have lots of copies of the same pair in the representation of a function seems a bit wasteful.
} 


$$
\begin{aligned}
& \operatorname{SAT}(c, \text { sat }): \leftrightarrow \forall x \forall a \leqslant c\left[\left(\operatorname{form}^{c}(a) \wedge \operatorname{ass}(c, x, a)\right) \rightarrow\right. \\
& \left\{\operatorname { s a t } ( x , a ) \leftrightarrow \left\langle\exists b<c\left(\llbracket P \rrbracket^{c}(b, a) \wedge P(x(b)) \vee\right.\right.\right. \\
& \exists b_{0}, b_{1}<c\left(\llbracket Q \rrbracket^{c}\left(b_{0}, b_{1}, a\right) \wedge Q\left(x\left(b_{0}\right), x\left(b_{1}\right)\right)\right) \vee \\
& \llbracket \top \rrbracket^{c}(a) \vee \llbracket \perp \rrbracket^{c}(a) \vee \\
& \exists b<c\left(\llbracket \neg \rrbracket^{c}(b, a) \wedge \neg \operatorname{sat}(x, b)\right) \vee \\
& \exists b_{0}, b_{1}<c \exists x_{0}, x_{1}\left(x_{0} \subseteq x \wedge x_{1} \subseteq x \wedge \operatorname{ass}\left(c, x_{0}, b_{0}\right) \wedge\right. \\
& \operatorname{ass}\left(c, x_{1}, b_{1}\right) \wedge\left(\llbracket \wedge \rrbracket^{c}\left(b_{0}, b_{1}, a\right) \wedge\right. \\
& \exists b_{0}, b_{1}<a\left(\llbracket \forall \rrbracket^{c}\left(b_{0}, b_{1}, a\right) \wedge\right. \\
& \left.\operatorname{sat}\left(x_{0}, b_{0}\right) \wedge \operatorname{sat}\left(x_{1}, b_{1}\right)\right) \vee \\
& \left.\left.\left.\left.\forall w\left(x\left[b_{0}\right] w \rightarrow \operatorname{sat}\left(w, b_{1}\right)\right)\right)\right\rangle\right\}\right]
\end{aligned}
$$

Figure 1. Definition of "sat is a satisfaction predicate below $c$ ".

form ${ }^{b}(\ulcorner C\urcorner), \mathbb{\llbracket} \forall \rrbracket^{b}(\ulcorner v\urcorner,\ulcorner C\urcorner,\ulcorner A\urcorner)$ and $\mathrm{fv}^{b}(\ulcorner v\urcorner)$. The induction hypothesis gives us:

(†) $V \vdash \operatorname{ass}(b, w,\ulcorner C\urcorner) \rightarrow\left(B(b, w,\ulcorner C\urcorner) \leftrightarrow C\left(w \star\ulcorner v\urcorner, w \star\left\ulcorner v_{0}\right\urcorner, \ldots, w \star\left\ulcorner v_{k-1}\right\urcorner\right)\right)$.

We work in $V$. Suppose $B(b, x,\ulcorner A\urcorner)$. It follows that $\forall w x[\ulcorner v\urcorner] w B(b, w,\ulcorner C\urcorner)$. Consider any $z$. Since $n>2$, we have that $\underline{2}$ exists. So we have unordered pairing. Hence, we can construct an ordered pair $p$ such that pair $(\ulcorner v\urcorner z, p)$. Since the number of free variables in $C$ is smaller than $\ulcorner A\urcorner$ and $\ulcorner A\urcorner$ exists, we can, using repeated adjunction, find a $y$ such that on $\left\ulcorner v_{i}\right\urcorner$, for $i<k$, we have $y \star\left\ulcorner v_{i}\right\urcorner=x \star\left\ulcorner v_{i}\right\urcorner$, and $y \star\ulcorner v\urcorner=z$. It is easy to see that ass $(b, y,\ulcorner C\urcorner)$ and $x[\ulcorner v\urcorner] y$. By (†), we find $C\left(y \star\ulcorner v\urcorner, y \star\left\ulcorner v_{0}\right\urcorner, \ldots, y \star\left\ulcorner v_{k-1}\right\urcorner\right)$. We may conclude that

$$
C\left(z, x \star\left\ulcorner v_{0}\right\urcorner, \ldots, x \star\left\ulcorner v_{k-1}\right\urcorner\right) .
$$

Since $z$ was arbitrary, it follows that $\forall z C\left(z, x \star\left\ulcorner v_{0}\right\urcorner, \ldots, x \star\left\ulcorner v_{k-1}\right\urcorner\right)$, in other words, $A\left(x \star\left\ulcorner v_{0}\right\urcorner, \ldots, x \star\left\ulcorner v_{k-1}\right\urcorner\right)$.

Conversely, suppose $A\left(x \star\left\ulcorner v_{0}\right\urcorner, \ldots, x \star\left\ulcorner v_{k-1}\right\urcorner\right)$. This means:

$$
\forall z C\left(z, x \star\left\ulcorner v_{0}\right\urcorner, \ldots, x \star\left\ulcorner v_{k-1}\right\urcorner\right) .
$$

Consider a $y$ with $x[\ulcorner v\urcorner] y$. We find: $C\left(y \star\ulcorner v\urcorner, y \star\left\ulcorner v_{0}\right\urcorner, \ldots, y \star\left\ulcorner v_{k-1}\right\urcorner\right)$. Hence, by (†), $B(b, y,\ulcorner C\urcorner)$. Thus, we find that, for all $y$ with $x[\ulcorner v\urcorner] y$, we have $B(b, y,\ulcorner C\urcorner)$. It follows that $B(b, x,\ulcorner A\urcorner)$.

Theorem 5.2. Let $K$ be any direct interpretation of $\mathrm{TS}_{0}$ in $U$, possibly with free parameters $z_{0}, \ldots, z_{p-1}$. Fix any $U$-formula $A(b, x, a, \vec{z})$. Then, for some $n$, we have: $U+\operatorname{SAT}(b, A) \vdash \bigvee_{i \leqslant n} b=\underline{i}$.

Proof. By Lemma 3.5, for some $n$, and for some formula $B \vec{v}$ with Gödel number $m$ :

$$
U+\underline{n} \downarrow \vdash B \vec{y} \leftrightarrow \exists a, s\left(\operatorname{ass}(b, s, a) \wedge \underline{m}=a \wedge \bigwedge_{i<p} s \star\left\ulcorner z_{i}\right\urcorner=z_{i} \wedge \neg A(s, a, \vec{z})\right) .
$$

We take $n$ so big that also $V+\underline{n} \downarrow \vdash$ form $^{\underline{n}}(\underline{m})$. 
Reason in $U$. Suppose $\operatorname{SAT}(b, A(x, a, \vec{z}))$. Suppose $\underline{n} \leqslant b$. Then we have, by Theorem 5.1,

$$
\begin{aligned}
B \vec{z} & \leftrightarrow \quad \exists a, s\left((\underline{m}=a)^{M} \wedge \bigwedge_{i<p} s\left(\left\ulcorner z_{i}\right\urcorner\right)=z_{i} \wedge \neg A(s, a, \vec{z})\right) \\
& \leftrightarrow \neg B \vec{z} .
\end{aligned}
$$

We may conclude, by Lemma 3.1(10), that $U+\operatorname{SAT}(b, A) \vdash \bigvee_{i<n} b=\underline{i}$.

\section{Proof of The Main Result}

Consider any theory $U$. Let $\alpha$ be a $\Sigma_{1}^{0}$-formula defining the set of Gödelnumbers of the axioms of $U$. We assume that $\alpha^{n}(m)$ implies form ${ }^{n}(m)$. We define our scheme as follows:

- $\mathcal{S}_{\alpha}:=\wedge \mathrm{TS}_{0} \rightarrow \forall a, b\left(\left(\alpha^{b}(a) \wedge \mathrm{SAT}(b\right.\right.$, sat $\left.\left.)\right) \rightarrow \exists s(\operatorname{empty}(s) \wedge \operatorname{sat}(b, s, a))\right)$.

We find that $U$ satisfies the scheme.

Theorem 6.1. Suppose the axiom set of $U$ is given by the $\Sigma_{1}^{0}$-formula $\alpha$. Then, $U$ validates $\mathcal{S}_{\alpha}$.

Proof. Consider any direct interpretation $K$ (possibly with free parameters $\vec{z}$ ) for the language of $\mathrm{TS}_{0}$. In the following we will suppress $K$. Let $A:=A(b, s, a, \vec{z})$ be any formula. Let $V:=U+\mathrm{TS}_{0}+\alpha^{b}(a)+\operatorname{SAT}(b, A)$. By Theorem 5.2, we find, for some $n, V \vdash \bigvee_{j<n} b=\underline{j}$. It follows that $V \vdash \bigvee_{i<n} a=\underline{i}$. Fix $i, j<n$. If we have not $\alpha^{j}(i)$. Then, by Lemma 3.3, we find $V \vdash \neg \alpha \underline{\underline{j}}(\underline{i})$. So, it follows that $V \vdash \neg(a=\underline{i} \wedge b=\underline{j})$ and a fortiori, $V+a=\underline{i}+b=\underline{j} \vdash \exists s(\operatorname{empty}(s) \wedge \mathrm{A}(b, s, a)))$. If we have $\alpha^{i}(j)$. Then we find, by Lemma 3.3, $V \vdash \alpha \underline{\underline{j}}(\underline{i})$. Suppose $i$ is the Gödelnumber of $C$. Since, $U \vdash C$, we find, by Theorem 5.1, $V+a=\underline{i}+b=\underline{j} \vdash$ $\exists s(\operatorname{empty}(s) \wedge A(b, s, a)))$. So, we obtain $V \vdash \exists s(\operatorname{empty}(s) \wedge A(b, s, a)))$.

Theorem 6.2. Consider a theory $U$ whose axiom set is given by the $\Sigma_{1}^{0}$-formula $\alpha$. We suppose that we have a direct interpretation $M$, of $\mathrm{VS}_{2}$ in $U$. We write $\left[\mathcal{S}_{\alpha}\right]$, for the set of instances of $\mathcal{S}_{\alpha}$ in the signature of $U$. Then, $\mathrm{VS}_{2}^{M}+\left[\mathcal{S}_{\alpha}\right]$ proves the same theorems as $U$.

Proof. The fact that $U$ proves all principles of $V:=\mathrm{VS}_{2}^{M}+\left[\mathcal{S}_{\alpha}\right]$ is immediate by Theorem 6.1. We prove the converse.

Consider an axiom $A$ of $U$ and suppose $\alpha^{n}(\ulcorner A\urcorner)$. Theorem 4.1 provides a direct interpretation $K$ of $\mathrm{TS}_{n}$ in $\mathrm{VS}_{2}$. So, $L:=K \circ M$ is a direct interpretation of $\mathrm{TS}_{n}$ in $U$. We define a satisfaction predicate $B(x, c)$, for all formulas $C$, such that form ${ }^{n}(C)$, just by making it the disjunction of all formulas:

- $(c=\ulcorner C\urcorner) \wedge \operatorname{ass}(\underline{n}, x,\ulcorner C\urcorner) \wedge C\left(x \star\left\ulcorner v_{0}\right\urcorner, \ldots, x \star\left\ulcorner v_{k-1}\right\urcorner\right)$, where $C=C\left(v_{0}, \ldots, v_{k-1}\right)$ and form ${ }^{n}(\ulcorner C\urcorner){ }^{3}$

We find in $V$ that, under interpretation $L$, we have $\operatorname{SAT}(\underline{n},\ulcorner B\urcorner)$. Let $s$ be any empty set. By $\mathcal{S}_{\alpha}$, instantiated via $L$ for the signature of $\mathrm{VS}_{n}$ and by substituting $B$ for sat, we find: $V \vdash B(\underline{n}, s,\ulcorner A\urcorner)$. Hence, $V \vdash A$.

\footnotetext{
${ }^{3}$ Of course, this is a very inefficient way, in terms of size, to make such a predicate. One can clearly do much better.
} 
We note that the result works both for the case that we consider closed schemes or schemes with parameters.

Inspection of the methods used shows that the result is verifiable in EA. It follows that $\mathrm{EA} \vdash \operatorname{con}(\alpha) \leftrightarrow \operatorname{con}\left(\mathrm{VS}_{2}^{M}+\left[\mathcal{S}_{\alpha}\right]\right)$.

\section{Comprehension Principles}

Schemes can be condensed into single sentences in the presence of suitable comprehension principles. We briefly consider what happens in two cases: First Order Comprehension or FOC and Predicative Comprehension or PC.

7.1. First Order Comprehension. Let us start with a pair theory $U$ which is axiomatized by a scheme $\mathcal{S}_{0}$. We first show that $\mathcal{S}_{0}$ can be replaced by a unary scheme $\mathcal{S}$, i.e., a scheme in which only unary schematic predicates occur. We note that in $\mathrm{VS}_{2}$ we can define Wiener-Kuratowski pairing. Let pair $(x, y, z)$ be:

$$
\begin{aligned}
& \exists u, v(\forall w(w \in z \leftrightarrow(w=u \vee w=v)) \wedge \\
& \left.\forall w^{\prime}\left(w^{\prime} \in u \leftrightarrow w^{\prime}=x\right) \wedge \forall w^{\prime \prime}\left(w^{\prime \prime} \in v \leftrightarrow\left(w^{\prime \prime}=x \vee w^{\prime \prime}=y\right)\right)\right) .
\end{aligned}
$$

Using pair we can define $n$-tuple for standard $n$. Also we can define the associated projection functions. We can verify the desired properties in $\mathrm{VS}_{2}$. We assign to every schematic predicate $P$ a unary schematic predicate $X_{P}$. Consider an occurrence of a schematic predicate $P\left(x_{0}, \ldots, x_{n-1}\right)$ in $\mathcal{S}_{0}$. We replace this occurrence by $\exists s\left(n\right.$-tuple $\left.(s) \wedge(s)_{0}=x_{0} \wedge \ldots \wedge(s)_{n-1}=x_{n-1} \wedge s \in X_{P}\right)$. Similarly, for all other occurrences of schematic predicates in $\mathcal{S}_{0} \cdot{ }^{4}$ Suppose in the original scheme we would substitute $A \vec{x}$ for $P \vec{x}$. Clearly, we will obtain the same effect by substituting $\left(n\right.$-tuple $\left.(s) \wedge \exists x_{0}, \ldots, x_{n-1}\left((s)_{0}=x_{0} \wedge \ldots \wedge(s)_{n-1}=x_{n-1} \wedge A \vec{x}\right)\right)$ for $X_{P}$.

We suppose that $U$ is axiomatized by a unary scheme $\mathcal{S}$. We extend $U$ with a new sort of classes $\mathfrak{c}$, identity on classes and a binary relation $\in$ of type $\mathfrak{o c}$. Here $\mathfrak{o}$ is the sort of the original objects of $U$. We use the variables $X, Y, Z, \ldots$ to range over classes. The theory $\mathcal{S}^{\text {foc }}$ in the extended signature is defined as follows.

$\mathcal{S}^{\text {foc }} 1 . \vdash \forall \vec{y} \exists X \forall x(x \in X \leftrightarrow A x \vec{y})$,

where $A$ is a formula from the original language of $U$.

$\mathcal{S}^{\text {foc }} 2 . \vdash \forall X, Y(X=Y \leftrightarrow \forall z(z \in X \leftrightarrow z \in Y))$.

$\mathcal{S}^{\text {foc }} 3 . \vdash \forall \vec{X} \mathcal{S}^{\star} \vec{X}$

where $\mathcal{S}^{\star}$ is the result of replacing all occurrences of schematic predicates in $\mathcal{S}$ by class variables in the obvious way, e.g. $X x$ by $x \in X$.

The next theorem gives us the obvious connection between $U$ and $\mathcal{S}^{\text {foc }}$.

Theorem 7.1. Suppose $U$ is a pair theory, axiomatized by a unary scheme $\mathcal{S}$. Clearly, $\mathcal{S}^{\text {foc }}$ is a conservative extension of $U$. Moroever $U$ locally $\mathfrak{o}$-directly interprets $\mathcal{S}^{\text {foc }}$.

Proof. We work in $U$. Suppose we want to have comprehension for the formulas $A_{i} x \vec{y}_{i}$, for $i=0, \ldots, k-1$, where the length of $\vec{y}_{i}$ is $n_{i}$. We define:

$$
\begin{gathered}
\text { - } \mathbf{i}(v): \leftrightarrow \exists v_{0}, \ldots, v_{i-1}\left(\forall w w \notin v_{0} \wedge \forall w\left(w \in v_{1} \leftrightarrow w=v_{0}\right) \wedge \ldots\right. \\
\text { - } \operatorname{class}_{i}(v): \leftrightarrow\left(n_{i}+1\right)-\operatorname{tuple}(v) \wedge \mathbf{i}\left((v)_{0}\right) .
\end{gathered}
$$

\footnotetext{
${ }^{4}$ If a schematic predicate in $\mathcal{S}_{0}$ is 0 -ary, we introduce a dummy variable.
} 
- $\operatorname{class}(v): \leftrightarrow \bigvee_{i<k} \operatorname{class}_{i}(v)$.

- $x \in v: \leftrightarrow \operatorname{class}(v) \wedge \bigvee_{i<k}\left(\mathbf{i}\left((v)_{0}\right) \wedge A_{i}\left(x,(v)_{1}, \ldots,(v)_{n_{i}}\right)\right)$.

- $v={ }_{\mathfrak{c}} w: \leftrightarrow \operatorname{class}(v) \wedge \operatorname{class}(w) \wedge \forall x(x \in v \leftrightarrow x \in w)$.

We take class as the domain for the sort of classes, $\in$ in the obvious role and $=_{\mathfrak{c}}$ as identity of classes. It is easy to check that this interpretation has the desired properties.

An interpretation is $\mathfrak{o}$-direct if it interprets the sort $\mathfrak{o}$ as the sort $\mathfrak{o}$ and if it is unrelativized for $\mathfrak{o}$ and sends $\mathfrak{o}$-identity to itself. We have an interesting connection between $\mathrm{VS}_{2}^{\text {foc }}$ and VS.

Theorem 7.2. We have $\mathrm{VS}_{2}^{\text {foc }} \triangleright_{\mathfrak{o} \text {-dir }} \mathrm{VS}$.

Proof. We work in $\mathrm{VS}_{2}^{\text {foc }}$. We first define Wiener-Kuratowski pairing. We work with ordered pairs. To give the heuristic, let's ignore for a moment the fact that our ordered pairing is not necessarily functional. The basic idea is to code e.g. the set consisting of $a, b, c$ as $\langle\langle\langle 0, a\rangle, b\rangle, c\rangle$, where 0 is a non-pair. Now forget about functionality again. We define:

- $\mathrm{dc}(Y): \leftrightarrow \forall u, v, p((\operatorname{pair}(u, v, p) \wedge p \in Y) \rightarrow u \in Y)$,

(We will also write $Y: \mathrm{dc}$ for $\mathrm{dc}(Y)$.)

- $x \in^{\star} y: \leftrightarrow \forall Y: \mathrm{dc}(y \in Y \rightarrow \exists w, q(\operatorname{pair}(w, x, q) \wedge q \in Y))$.

Consider any non-pair $z$. We clearly have $\{z\}$ :dc. If we would have $x \in z$, then, for some pair $q$, we would have that $q$ is in the class $\{z\}$, quod non. So $z$ is an empty $\star$-set. This gives us $\mathrm{VS}_{0}$.

We have the following insight $(\dagger)$ : Consider any $x$ and $y$. Pick any $z$ with pair $(y, x, z)$. Clearly, $x \in^{\star} z$. Moreover, we find $y \subseteq^{\star} z$. For, suppose $u \in^{\star} y, \mathrm{dc}(Y)$ and $z \in Y$. We find $y \in Y$, and, hence, for some $\bar{w}$ and $q$, $\operatorname{pair}(w, u, q)$ and $q \in Y$. So $u \in z$.

We now show $\mathrm{VS}_{n}$ be induction on $n$. Let $x_{0}, \ldots, x_{n-1}$ be given. We construct a sequence of $\star$-sets $z_{0}, \ldots, z_{n}$ as follows. Let $z_{0}$ be any non-pair. We take $z_{i+1}$ to be an object such that pair $\left(z_{i}, x_{i-1}, z_{i+1}\right)$. We note, by $(\dagger)$, that $x_{j} \in^{\star} z_{i}$, for all $j<i$.

Let $Y_{i}$ be the class $\left\{z_{0}, \ldots, z_{i}\right\}$. We easily verify $Y_{i}$ is downwards closed. Clearly for any $u$ not among $x_{0}, \ldots x_{i-1}$, we find that no $v$ such that pair $(w, u, v)$, for some $w$, is in $Y_{i}$. On the other hand $z_{i}$ is in $Y_{i}$. It follows that $u \notin^{\star} z_{i}$. Ergo, for all $u$, $u \in^{\star} z_{i}$ iff $u=x_{0}$ or $\ldots$ or $u=x_{i-1}$.

Theorem 7.2 suggests the following questions.

Open Question 7.3. Suppose $U$ is a pair theory axiomatized by a scheme $\mathcal{S}$. Do we have, for any $W$ with $W \triangleright U \triangleright_{\text {loc }} W$, that $\mathcal{S}^{\text {foc }} \triangleright W ?^{5}$

Open Question 7.4. Do we have VS $\triangleright \mathrm{VS}_{2}^{\text {loc }}$ ?

7.2. Predicative Comprehension. In our paper [Vis09a], we elaborate the equation "consistency = predicative comprehension". In that context, Vaught's result provides on way of articulating what predicative comprehension for infinitely axiomatized RE pair theories means.

Consider a pair theory $U$ that is axiomatized by a scheme $\mathcal{S}$. As before we may assume that $\mathcal{S}$ only contains unary predicate variables. We extend the language

\footnotetext{
${ }^{5}$ We know that this holds when $U$ is a sequential theory.
} 
of $U$ with class variables. The theory $\mathcal{S}^{\mathrm{pc}}$ in the extended signature is defined as follows.

$\mathcal{S}^{\mathrm{pc}} 1 . \vdash \forall \vec{y} \exists X \forall x(x \in X \leftrightarrow A x \vec{y} \vec{Y})$, where $A$ is a formula that contains no bound class variables and that does not contain $X$.

$\mathcal{S}^{\mathrm{pc}} 2 . \vdash \forall X, Y(X=Y \leftrightarrow \forall z(z \in X \leftrightarrow z \in Y))$.

$\mathcal{S}^{\mathrm{pc}} 3 . \vdash \forall \vec{X} \mathcal{S}^{\star} \vec{X}$, where $\mathcal{S}^{\star}$ is the result of replacing all occurrences of schematic predicates in $\mathcal{S}$ by class variables.

Clearly, $\mathcal{S}^{\mathrm{pc}}$ is a conservative extension of $U$. One can show that $\mathcal{S}^{\mathrm{pc}}$ is finitely axiomatizable. In case that $U$ is sequential one can show that $(\mathrm{Q}+\operatorname{con}([\mathcal{S}])) \equiv \mathcal{S}^{\mathrm{pc}}$. The details of this are explained in [Vis09b] and [Vis09a]. So, we note that, by the Second Incompleteness Theorem, $\mathcal{S}^{\mathrm{pc}}$ is really stronger than $U$.

We may apply Vaught's result, verifiably in EA, to any consistent sequential theory RE theory $U$, say axiomatized using $\alpha$. This gives us:

$$
\mathrm{EA} \vdash(\mathrm{Q}+\operatorname{con}(\alpha)) \equiv \mathcal{S}_{\alpha}^{\mathrm{pc}} \text {. }
$$

However it is easy to see that, for any true $\Pi_{1}^{0}$-sentence $P$, we can find an axiomatization $\beta$ of $U$ such that $(\mathrm{Q}+\operatorname{con}(\beta)) \triangleright(\mathrm{Q}+P)$. Thus, $\mathcal{S}_{\beta}{ }^{\mathrm{pc}}$ can be made arbitrarily strong, when $\beta$ ranges over schemes for $U$. This illustrates that a scheme is 'intensional' from the point of view of consistency strength.

We end with a question.

Open Question 7.5. Do we have $(\mathrm{Q}+\operatorname{con}([\mathcal{S}])) \equiv \mathcal{S}^{\mathrm{pc}}$, for pair theories $U$ axiomatized by $\mathcal{S}$ ?

\section{REFERENCES}

[CR01] P. Cégielski and D. Richard. Decidability of the natural integers equipped with the Cantor pairing function and successor. Theoretical Computer Science, 257, 1-2:51-77, 2001.

[FR79] J. Ferrante and C.W. Rackoff. The computational complexity of logical theories, volume 718 of Lecture Notes in Mathematics. Springer, Berlin, 1979.

[Ten] R.L. Tenney. Decidable pairing functions. Unpublished.

[Vau67] R.A. Vaught. Axiomatizability by a schema. The Journal of Symbolic Logic, 32(4):473479, 1967.

[Vis06] A. Visser. Categories of Theories and Interpretations. In Ali Enayat, Iraj Kalantari, and Mojtaba Moniri, editors, Logic in Tehran. Proceedings of the workshop and conference on Logic, Algebra and Arithmetic, held October 18-22, 2003, volume 26 of Lecture Notes in Logic, pages 284-341. ASL, A.K. Peters, Ltd., Wellesley, Mass., 2006.

[Vis08] A. Visser. Pairs, sets and sequences in first order theories. Archive for Mathematical Logic, 47(4):299-326, 2008.

[Vis09a] A. Visser. Can we make the Second Incompleteness Theorem coordinate free. Journal of Logic and Computation, 2009. doi: 10.1093/logcom/exp048.

[Vis09b] A. Visser. The predicative Frege hierarchy. Annals of Pure and Applied Logic, 160(2):129-153, 2009. doi: 10.1016/j.apal.2009.02.001.

Department of Philosophy, Utrecht University, Heidelberglaan 8, 3584 CS Utrecht, The NetherLANDS

E-mail address: Albert.Visser@phil.uu.nl 